\title{
Invasive meningococcal disease without meningitis: a forgotten diagnosis
}

This article was published in the following Dove Press journal: International Medical Case Reports Journal

\author{
Saqib Walayat ${ }^{\prime}$ \\ Nooreen Hussain' \\ Abdullah H Malik' \\ Elsa Vazquez-Melendez' \\ Bhagat S Aulakh² \\ Teresa Lynch' \\ 'Department of Internal Medicine, \\ University of Illinois College of \\ Medicine at Peoria, Peoria, IL, USA; \\ ${ }^{2}$ Department of Pulmonary/Critical \\ Care Medicine, University of Illinois \\ College of Medicine at Peoria, Peoria, \\ IL, USA
}

Correspondence: Saqib Walayat

Department of Internal Medicine, University of Illinois College of Medicine at Peoria, 530 NE Glen Oak Ave, Peoria, IL 61637, USA

Tel +l 650787748

Email Saqibwalayat@gmail.com

\begin{abstract}
Neisseria meningitidis, a Gram-negative diplococcus, is an uncommon cause of pneumonia. There have been only about 344 cases reported worldwide from 1906 to 2015. To our knowledge, there have been only 3 cases reported in the USA in the past 2 decades. We present a case of a 72-year-old male with a past medical history of severe COPD, obstructive sleep apnea, and stage I lung cancer status post-stereotactic body radiation therapy 1 year ago, who was admitted with a 6-day history of productive cough with yellowish sputum, shortness of breath, extreme myalgias, and fatigue. Chest X-ray revealed an infiltrative process in the left lower lung field and left-sided pleural effusion. Blood cultures grew beta-lactamase-negative $N$. meningitidis after 24 hours. Our patient was initially treated with broad-spectrum antibiotics, which were later switched to amoxicillin to complete a total of 14 days of antibiotics. Diagnosing meningococcal pneumonia requires a high level of suspicion, as sputum cultures may be falsely positive due to asymptomatic carriage of the organism in the upper respiratory tract in up to $10 \%$ of outpatient population. We highlight this case as early recognition and treatment is critical. The case fatality rate for $N$. meningitidis pneumonia has been reported to be higher compared with meningococcal meningitis.
\end{abstract}

Keywords: Neisseria meningitidis, pneumonia, invasive meningococcal pneumonia, sepsis

\section{Introduction}

Neisseria meningitidis, a Gram-negative diplococcus, is an uncommon cause of pneumonia. It is feared for its rapid progression and high mortality rate, and is an important cause of sepsis and meningitis worldwide. After meningitis, the second most common end-organ presentation is meningococcal pneumonia, which continues to remain underreported. ${ }^{1}$ The exact incidence of meningococcal pneumonia remains unknown but is estimated to be $5 \%-15 \%$ in patients with invasive meningococcal disease. ${ }^{1,2}$ There have been only about 344 case reports of meningococcal pneumonia between 1906 and 2015. ${ }^{1}$ The diagnosis requires a high level of suspicion and, to our knowledge, there have been only 3 cases reported in the USA in the past 2 decades (since 1998), further indicating that this disease is likely underreported and underestimated. ${ }^{1-5}$ Invasive meningococcal disease is of major public health importance due to its global distribution, epidemic potential, and fulminant clinical manifestations. ${ }^{6}$ Recognition of invasive meningococcal disease and early treatment is imperative in preventing complications or death and preventing spread of the disease to others. 


\section{Case report}

A 72-year-old male with a past medical history of hypertension, severe COPD, obstructive sleep apnea, stage IV prostate cancer on enzalutamide, stage I lung cancer status post-stereotactic body radiation therapy 1 year earlier, and chronic immune-mediated thrombocytopenia initially came to the emergency department with a 6-day history of productive cough with yellowish sputum, shortness of breath, severe myalgia, fatigue, and delirium. The patient denied fevers, chills, headache, skin rash, recent travel, or sick contacts. Vital signs on admission were significant for blood pressure of $92 / 50 \mathrm{mmHg}$, heart rate of 134 beats per minute, temperature of $38.3^{\circ} \mathrm{C}$, respiratory rate of 21 breaths per minute, and oxygen saturation of $86 \%$ on room air. Physical examination revealed diffuse expiratory wheezing in all lung fields. Initial lab work was significant for a white blood cell count of $31,000 /$ $\mu \mathrm{L}$ (neutrophils $72 \%$, lymphocytes $2 \%$, and bands $15 \%$ ), lactic acid of $3.1 \mathrm{mmol} / \mathrm{L}$, hemoglobin of $12.4 \mathrm{~g} / \mathrm{dL}$, platelet count of $26,000 / \mu \mathrm{L}$ (baseline around $30,000-40,000 / \mu \mathrm{L}$ ), creatinine of $2.5 \mathrm{mg} / \mathrm{dL}$, creatine kinase of $76 \mathrm{U} / \mathrm{L}$, and procalcitonin of $35 \mathrm{ng} / \mathrm{mL}$. Chest X-ray showed an infiltrative process in the left lower lung field and left-sided pleural effusion (Figure 1). Sputum Gram stain showed Gram-negative diplococci in addition to Gram-positive cocci and Gram-negative bacilli. The patient was given $3 \mathrm{~L}$ of fluid in the emergency department and was initially started on ceftriaxone and azithromycin for community-acquired pneumonia. This was later switched to vancomycin and levofloxacin, considering the risk of severe sepsis secondary to pneumonia in an immunocompromised host. The patient's blood culture polymerase chain reaction (PCR) was positive for N. meningitidis; however, he did not have signs of meningitis and a lumbar puncture was not performed. Our patient's fever improved the next day of admission and his cough continued to improve throughout

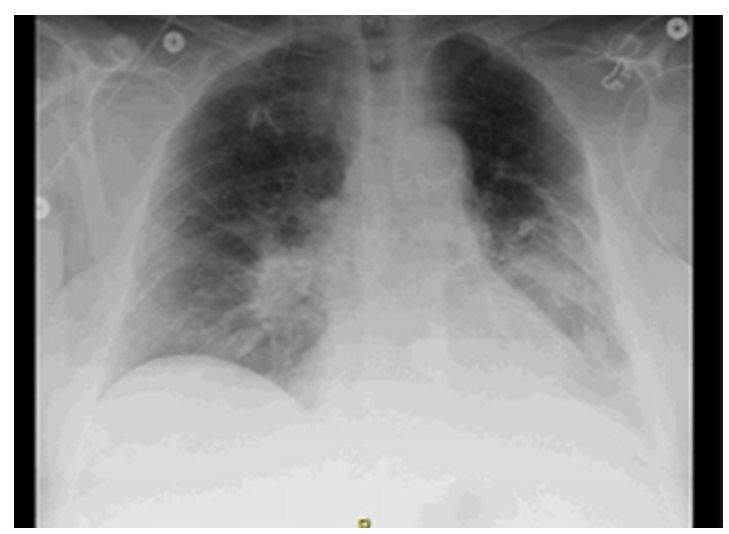

Figure I Chest X-ray showed an infiltrative process in the left lower lung field and a left sided pleural effusion. the admission. Subsequent respiratory cultures grew heavy mixed flora. Blood cultures grew $\beta$-lactamase-negative $N$. meningitidis after 24 hours. Antibiotics were initially switched to ceftriaxone for 5 days followed by amoxicillin $500 \mathrm{mg} 3$ times daily for a total of 14 days of antibiotics after sensitivity testing revealed the organism to be sensitive to penicillin, ceftriaxone, and levofloxacin, with minimum inhibitory concentrations (MICs) of $\leq 0.06, \leq 0.12$, and $\leq 0.03$, respectively. The department of health was notified, the patient was given Meningivax and Prevnar 13 prior to discharge, and close contacts were notified to take levofloxacin.

Written consent was obtained from the patient for the case details to be published.

\section{Discussion}

$N$. meningitidis pneumonia has been reported as early as the 1900 s when it was linked to the influenza pandemic. ${ }^{1}$ It is an asymptomatic colonizer in throat cultures of $10 \%$ of adult patients. ${ }^{3}$ Carriage rates increase progressively with age, from $0.71 \%$ to $3 \%$ in children $<4$ years of age and is highest in adolescents and young adults up to 25 years of age at $32 \%-37 \%$, and this has been proposed to be due to changes in social behavior with age. ${ }^{6,11}$ In non-epidemic settings, $\sim 10 \%$ of healthy individuals (including older age groups) at any time carry $N$. meningitidis in the upper airway. ${ }^{12}$ Increased risk of carriage includes military barracks, close contact or intimate kissing, the Hajj pilgrimage, damage to the nasopharyngeal mucosa from active or passive smoking, or from coinfection with influenza and Mycoplasma species. ${ }^{6}$ Risk factors for invasion and active infection include immunocompromised state, diabetes mellitus, COPD, malignancy, and human immunodeficiency virus infection. ${ }^{3}$ The pathogenesis that leads from colonization to invasive disease is not completely understood, but it is thought to be the result of meningococcal virulence factors, environmental conditions, and host susceptibility. ${ }^{6}$ One mechanism that is proposed is microaspiration of bacteria in the appropriate clinical settings and subsequently developing pneumonia. ${ }^{1}$ Another proposed mechanism is spreading to the lungs from the bloodstream as $23 \%$ of patients with positive blood cultures also have infiltrates on chest X-ray. ${ }^{1}$ It is possible that our patient could have been a colonizer, and then he had a silent aspiration event, leading to pneumonia and subsequent bacteremia. Serogroup Y (44\%) followed by W-135 (19\%) have been reported to be the most common isolates. The reason for these 2 being the more prevalent strains remains unknown. The prevalence of serogroup Y meningococcal disease has recently increased in the USA. ${ }^{5}$ Serogroup Y 
is also more likely than other serogroups to be associated with pneumonia. ${ }^{1}$ Hence, the prevalence of meningococcal pneumonia has probably increased, but it continues to remain underreported. Interestingly, from the review of case reports, it seems when one gets $N$. meningitidis pneumonia, he is less likely to get meningitis. No guidelines or evidence are found to support preemptive lumbar puncture or brain imaging in these patients without signs or symptoms of meningitis or encephalitis.

Meningococcal pneumonia has a bimodal distribution pattern, $<30$ and $>60$ years of age, with median being around 57.5 years. Presentation as pneumonia is more common in the elderly, and, in fact, in patients $>65$ years of age, pneumonia is the most common presentation of invasive meningococcal disease. ${ }^{1}$ The mean duration to symptoms and presentation of meningococcal pneumonia was 5.5 days. Fever and chills ( $65 \%$ of patients), pleuritic chest pain $(53.9 \%$ of patients), productive cough (31\% of patients), and shortness of breath ( $23 \%$ of patients) are the most common symptoms at presentation. ${ }^{3}$

Diagnosing meningococcal pneumonia requires a high level of suspicion as sputum cultures may be falsely positive due to asymptomatic carriage of the organism in the upper respiratory tract, while blood culture sensitivity rates have been reported as anywhere from $6 \%$ to $79 \% .{ }^{1}$ Blood chemistries are usually supportive of an acute inflammatory process with the presence of leukocytosis and increased acute phase protein. ${ }^{1}$ In our case report, procalcitonin was remarkably elevated in addition to the marked leukocytosis. Blood or pleural cultures that yield $N$. meningitidis establish a diagnosis with certainty. ${ }^{7}$ Real-time PCR assays have been reported to be highly sensitive and specific. ${ }^{7}$ They reduce turnaround time and help in making an early diagnosis such as in our case; however, they are not yet available everywhere. ${ }^{7}$ Chest X-ray usually shows a lobar pattern of involvement with the right lower lobe being reported to be more commonly involved. ${ }^{3}$ Pleural effusion, as reported in our case, has only been reported in $13 \%$ of cases of meningococcal pneumonia. ${ }^{1}$

For many years, penicillin $\mathrm{G}$ was the drug of choice for treating meningococcal infections, until strains with decreased susceptibility to penicillin were reported worldwide. The SENTRY program surveyed N. meningitidis isolates in North America from 1998 to 1999 and found a higher prevalence of decreased susceptibility to penicillin than previously reported. From the resistant strains isolated, serogroup Y comprised 24\%. Penicillin-resistant $N$. meningitidis infection in the USA was first reported in 2006 in a teenage girl who had presented with bilateral extensive pneumonia and empyema. Penicillin resistance is defined by a MIC of $\geq 0.5 \mu \mathrm{g} / \mathrm{mL}$ by The Clinical and Laboratory Standards Institute..$^{5}$ Third-generation cephalosporins continue to remain as the mainstay of treatment in the USA. Fever was reported to resolve within 3 days in $93 \%$ of patients (68 patients) with the advent of antimicrobial therapy. ${ }^{2}$ No definite guidelines exist with regard to glucocorticoid administration in meningococcal pneumonia and it is generally not recommended. ${ }^{1}$ Chemoprophylaxis is recommended for household or close contact exposure, those exposed to patients' oral secretions, and day-care contacts, with single dose fluoroquinolones such as ciprofloxacin or ofloxacin, or a single dose of azithromycin. ${ }^{8}$ Mortality rate is reported to be $\sim 9 \%{ }^{3}$

Currently, the US Centers for Disease Control and Prevention recommends vaccination for $N$. meningitidis only in patients with complement deficiencies, those with damaged spleen or splenectomy, those with HIV, those taking eculizumab, those traveling or living in countries where the disease is common, microbiologists routinely exposed to $N$. meningitidis, military recruits, those who are not up to date with this vaccine, and first-year college residents living in dorms or residence halls. No recommendations currently exist with regard to vaccinating the elderly or those who have been in close contact with affected individuals. ${ }^{9,10}$

The incidence of invasive meningococcal disease reported is between $5 \%$ and $15 \%$ of all patients with invasive meningococcal disease and is therefore the second most common non-systemic, end-organ manifestation of invasive meningococcal disease. ${ }^{1}$ A more accurate incidence is difficult to ascertain due to the uncertainty in establishing a cause of meningococcal pneumonia. Contributing factors are as follows: failure to consider meningococcus as a cause of pneumonia, nondistinctive clinical features of meningococcal pneumonia, and the estimated low rate of concomitant bacteremia (present in 15\%-26\% of pneumonia cases), and therefore, it is more likely to be underreported in the community. Isolation of this organism from the sputum is not a decisive criterion for diagnosis as asymptomatic individuals can also be carriers. Therefore, it remains essential to have a high clinical suspicion for meningococcal pneumonia, especially in patients who are immunocompromised and have sputum cultures showing Gram-negative diplococci. Early recognition and treatment is critical as the case fatality rate for $N$. meningitidis pneumonia has been reported to be higher compared with meningococcal meningitis $(16 \%$ versus $9 \%-14 \%$ ) and of equal importance is antibiotic prophylaxis for close contacts of these patients to prevent meningitis or septicemia. 


\section{Disclosure}

The authors report no conflicts of interest in this work.

\section{References}

1. Vossen M, Mitteregger D, Steininger C. Meningococcal pneumonia. Vaccine. 2016;34(37):4364-4370.

2. Romero-Gomez MP, Rentero Z, Paño JR, Mingorance J. Bacteraemic pneumonia caused by Neisseria meningitidis serogroup Y. Respir Med Case Rep. 2012;5:23-24.

3. Johnathan M Winstead, David S McKinsey, Sybil Tasker, Mary Ann De Groote, Larry M Baddour. Meningococcal pneumonia: characterization and review of cases seen over the past 25 years. Clin Infect Dis. 2000;30(1):87-94.

4. Peter Soltani, Terrence McGarry, Parth Rali. Primary meningococcal pneumonia without meningitis. Chest. 2012;142(4):243

5. Glikman D, Matushek SM, Kahana MD, Daum RS. Pneumonia and empyema caused by penicillin-resistant Neisseria meningitidis: a case report and literature review. Pediatrics. 2006;117(5):e1061-e1066.
6. Pace D, Pollard AJ. Meningococcal disease: clinical presentation and sequelae. Vaccine. 2012;30(Suppl 2):B3-B9.

7. McHugh MP, Gray SJ, Kaczmarski EB, Guiver M. Reduced turnaround time and improved diagnosis of invasive serogroup B Neisseria meningitidis and Streptococcus pneumoniae infections using a lyophilized quadruplex quantitative PCR. J Med Microbiol. 2015;64(11):1321-1328.

8. Memish ZA. Meningococcal disease and travel. Clin Infect Dis. 2002;34(1):84-90.

9. Baker CJ, Briere EZ, Clark TA, et al; Centers for Disease Control and Prevention (CDC). Prevention and control of meningococcal disease: recommendations of the Advisory Committee on Immunization Practices (ACIP). MMWR Recomm Rep. 2013;62(RR-2):1-28.

10. CDC-National Center for Health Statistics. Meningococcal vaccine: what everyone should know. Available from: https://www.cdc.gov/vaccines/vpd/mening/public/index.html\#should. Accessed May 19, 2017.

11. Stephens DS, Greenwood B, Brandtzaeg P. Epidemic meningitis, meningococcaemia, and Neisseria meningitides. Lancet. 2007;369(9580):2196-2210.

12. Yazdankhah SP, Caugant DA. Neisseria meningitidis: an overview of the carriage state. J Med Microbiol. 2004;53(Pt 9):821-832.
International Medical Case Reports Journal

\section{Publish your work in this journal}

The International Medical Case Reports Journal is an international, peer-reviewed open-access journal publishing original case reports from all medical specialties. Previously unpublished medical posters are also accepted relating to any area of clinical or preclinical science. Submissions should not normally exceed 2,000 words or

\section{Dovepress}

4 published pages including figures, diagrams and references. The manuscript management system is completely online and includes a very quick and fair peer-review system, which is all easy to use. Visit http://www.dovepress.com/testimonials.php to read real quotes from published authors.

Submit your manuscript here: https://www.dovepress.com/international-medical-case-reports-journal-journal 